\title{
Experimental and Theoretical Morphology of Single and Twinned Crystals of $\mathrm{Li}_{2} \mathrm{CO}_{3}$ (Zabuyelite)
}

\author{
Linda Pastero, Francesco Roberto Massaro, and Dino Aquilano* \\ Dipartimento di Scienze Mineralogiche e Petrologiche, Università degli Studi, via V. Caluso 35, \\ 10125 Torino, Italy
}

Received February 28, 2007; Revised Manuscript Received September 19, 2007

\begin{abstract}
Crystals of lithium carbonate (monoclinic $C 2 / c$ ) have been synthesized from aqueous solution at room temperature and pressure. Their observed growth morphology, essentially made by $\{001\},\{\overline{1} 01\}$, and $\{110\}$ forms, has been compared with the theoretical one calculated applying the Hartman-Perdok method and using a formal charge potential function. Theoretical crystal equilibrium shape has also been obtained and compared with that resulting from ab initio calculations. The growth shape of the usually observed 100 contact twins is interpreted as well, on the basis of the energetic misfit of the 100 twin law and the kinetic effect due to the presence of salient and re-entrant angles at the twin original composition plane.
\end{abstract}

\section{Introduction}

$\mathrm{Li}_{2} \mathrm{CO}_{3}$ crystals belong to the monoclinic system (space group $C 2 / c$ ), at variance with all other and most common rhombohedral and orthorhombic carbonates, as exhaustively described by Reeder. ${ }^{1}$ Lithium carbonate (mineral zabuyelite) is a poorly studied crystal because nothing is found about it in applied sciences and few findings are known in nature. $\mathrm{Li}_{2} \mathrm{CO}_{3}$ crystals were studied first by Mallard, ${ }^{2}$ who synthesized small individuals not exceeding half a millimeter in size, by either evaporation from aqueous solution at room temperature or slow cooling of the melted carbonate. He determined the axial ratios as $a: b: c=$ 1.672:1:1.224 and the $a^{\wedge} c$ angle equal to $65^{\circ} 35^{\prime}$. Moreover, he found that the contact 100 twins commonly occur and that the cleavage system is composed by the main, sharp 001 and the secondary, less sharp $\overline{1} 01$ plane.

Zabuyelite was discovered by Zheng and $\mathrm{Liu}^{3}$ in the Zabuye Salt Lake, Nagri, Tibet, where it occurs as micrometric crystals embedded in halite and as doubly terminated individuals (up to $1.2 \mathrm{~mm}$ in length) in near-shore precipitates. Zabuyelite was first recognized as a common and widespread solid state in spodumene-hosted fluid inclusions from three major Li-rich pegmatites ${ }^{4}$ using laser Raman spectroscopy. Moreover, zabuyelite rather than diomignite $\left(\mathrm{Li}_{2} \mathrm{~B}_{4} \mathrm{O}_{7}\right)$ was confirmed to be the major high-birifringence phase in fluid inclusions in spodumene from the Tanco pegmatite, as well as in three other large Li-rich pegmatites, Bikita and Kamatiwi in Zimbabwe and Kings Mountain in North Carolina. ${ }^{4}$

In spite of its low popularity, $\mathrm{Li}_{2} \mathrm{CO}_{3}$ crystals have been attracting a non-negligible interest since Rajam and $\mathrm{Mann}^{5}$ found that the morphology of $\mathrm{CaCO}_{3}$ crystals (calcite) growing from $\mathrm{Li}^{+}$doped aqueous solution changes from the cleavage $\{10 \overline{1} 4\}$ rhombohedron to the $\{0001\}$ pinacoid. More recently, Nefyodova et al. ${ }^{6}$ confirmed that the addition of lithium to hydrothermal growth solutions transforms the $\{10 \overline{1} 4\}$ calcite seeds in final crystals dominated by the $\{0001\}$ form. This experimental evidence, coupled with IR spectroscopic analysis ${ }^{5}$ and calculation on the segregation energy of lithium ions on the (0001) face, ${ }^{6-8}$ indicated that $\mathrm{Li}^{+}$ions cannot be segregated within the growing crystal bulk but can be randomly adsorbed on the $\{0001\}$ calcite surfaces and thus hindering their advancement rate.

Recently, systematic investigations on the nucleation and growth of calcite crystals from supersaturated aqueous

\footnotetext{
* Corresponding author. E-mail: dino.aquilano@unito.it.
}

solutions in the presence of variable concentrations of lithium allowed a new approach to understanding the complex relationship between lithium and calcite crystals morphology. ${ }^{9,10}$ An experimental "morphodrome", i.e., the diagram of supersaturation vs the $\left[\mathrm{Li}^{+}\right] /\left[\mathrm{Ca}^{2+}\right]$ concentration ratio, showed a continuous habit variation, from the dominant $\{10 \overline{1} 4\}$ form at a low $\left[\mathrm{Li}^{+}\right] /\left[\mathrm{Ca}^{2+}\right]$ ratio to the dominant $\{0001\}$ form at a high $\left[\mathrm{Li}^{+}\right] /\left[\mathrm{Ca}^{2+}\right]$ ratio. The morphological change was attributed to the formation of two-dimensional layers having the structure of the monoclinic $\mathrm{Li}_{2} \mathrm{CO}_{3}$ crystal, which is epitaxially adsorbed on the restructured $\{0001\}$ form of calcite. ${ }^{9}$ Furthermore, SEM and AFM observation proved that calcite crystals nucleated and grown from lithium-bearing gels and aqueous solutions show that $\{0001\}$-kinked and $\{01 \overline{1} 8\}$-stepped forms behave as flat (F) forms and that these transformations can be due to epitaxial relationships setting up between $\{001\},\{101\}$, and $\{100\}$ forms of $\mathrm{Li}_{2} \mathrm{CO}_{3}$ crystal and $\{0001\},\{01 \overline{1} 8\}$, and $\{10 \overline{1} 4\}$ forms of calcite. ${ }^{10}$

The systematic finding of these epitaxial relationships is the first reason why it is worth investigating the mutual relationships between the surface structures of the main crystallographic forms of the two structures. To do that, the experimental and theoretical morphologies of lithium carbonate are needed, whereas much information on the surface properties of calcite has already been acquired both in literature and from our previous researches.

Hence, we will confine our attention in this first paper on lithium carbonate crystals to their growth morphology and surface configuration. Thus, we will illustrate in detail the observed single and twinned $\mathrm{Li}_{2} \mathrm{CO}_{3}$ crystals obtained from aqueous solutions. Further, the theoretical equilibrium and growth morphology of the single crystals will be evaluated by preliminarily applying the Hartman and Perdok method ${ }^{11}$ for identifying the character of the $\{h k l\}$ forms, along with the possible profiles of their surfaces. As a second step, both specific surface energies and attachment energies will be calculated to draw the theoretical equilibrium and growth shapes of the crystal. Finally, a comparison will be done between the observed and calculated growth morphologies and an attempt will be made to explain the evolution of the growth shape of the 100 contact twin.

\section{Experimental Section}

There is hardly anything in the literature on methods for obtaining $\mathrm{Li}_{2} \mathrm{CO}_{3}$ crystals. As a matter of fact, solubility curves as a function of 

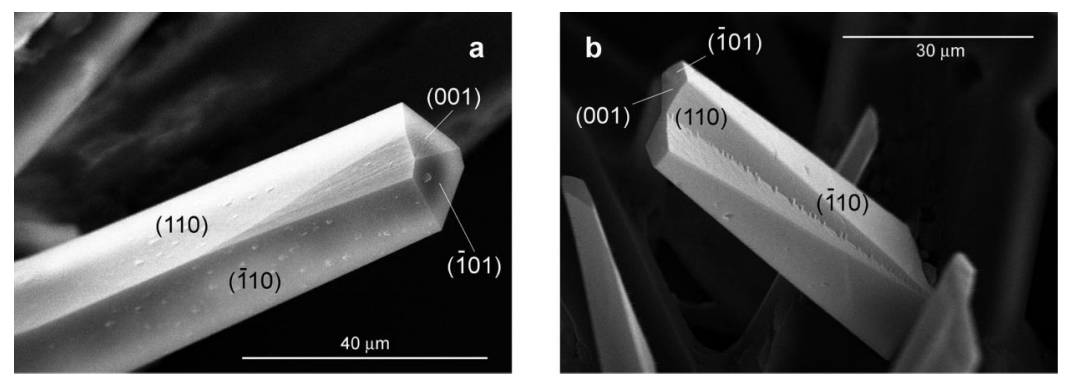

Figure 1. (a) SEM image of a $\mathrm{Li}_{2} \mathrm{CO}_{3}$ single crystal: the most important forms, $\{001\},\{10 \overline{1}\}$, and $\{110\}$, are shown, along with a small vicinal of the $\{010\}$ form. (b) Contact 100 twin where the re-entrant angle between the $\{110\}$ faces of the parent and twinned individuals is progressively filled from the beginning of growth.

temperature and $\mathrm{pH}$ needed for drawing growth morphodromes do not exist. Thus, the experimental procedures described in this paper will be inevitably empirical and confined to precipitate well-shaped crystals suitable for preliminary morphological studies; the quantitative investigations on the relationships between morphology and growth parameters are reserved for future works.

First, we synthesized $\mathrm{Li}_{2} \mathrm{CO}_{3}$ crystals at constant temperature (20 ${ }^{\circ} \mathrm{C}$ ) and ambient pressure, either by evaporation of saturated solution or by mixing two solutions containing counterions in variable amounts. Besides, some precipitations have been obtained from $\mathrm{Li}_{2} \mathrm{CO}_{3}$ solutions supersaturated by means of $\mathrm{CO}_{2}$ bubbling. Finally, well-formed crystals have been grown by slowly dripping a $\mathrm{LiOH}$ solution into a fresh carbonated water, obtained simply by bubbling $\mathrm{CO}_{2}$ into distilled water. We used analytical grade reagents (Riedel-de $\mathrm{Haën} \mathrm{LiCl}, \mathrm{LiOH}$, $\mathrm{Na}_{2} \mathrm{CO}_{3}$, and $\mathrm{NaHCO}_{3}$ ) to obtain mother solutions to be diluted and we worked both in pure solution and in the presence of ethyleneditrilotetra-acetic acid disodium salt (EDTA, Merk Titriplex III) to avoid the precipitation of $\mathrm{CaCO}_{3}$ as a byproduct of impure reagents.

Evaporation experiments were performed with a $\mathrm{LiCl}: \mathrm{NaHCO}_{3}$ molar ratio equal to 0.6 and Titriplex III ranging from zero to $0.5 \mathrm{M}$.

Experiments done by mixing two solutions were carried out in the absence of specific impurities in two ways: (a) by combining $\mathrm{LiCl}$ solutions ranging from $1.2 \times 10^{-2} \mathrm{M}$ to $6 \times 10^{-4} \mathrm{M}$ and $\mathrm{NaHCO}_{3}$ solutions ranging from 0.1 to $1 \mathrm{M}$ until the nucleation was reached; (b) by combining equimolar $\mathrm{LiCl}$ solutions and either $\mathrm{Na}_{2} \mathrm{CO}_{3}$ or $\mathrm{NaHCO}_{3}$ solutions to check, at least qualitatively, the effect of $\mathrm{pH}$ on nucleation and growth processes.

In the experiments carried out in pure solutions, distilled water was saturated with respect to the $\mathrm{CO}_{2}$ until acid $\mathrm{pH}$ values were reached; a $\mathrm{LiOH}$ solution was then dripped in the reactor until the final concentration was $0.52 \mathrm{M}$. Conductivity and $\mathrm{pH}$ values were monitored in real time by means of a DuraProbe four-electrode conductivity cell (with a cell constant of $0.475 \mathrm{~cm}^{-1}$ ) and a Thermo Orion 4-Star Benchtop pH/ Conductivity Meter equipped with a Orion 91-09 Triode 3-in-1 pH/ ATC probe.

The obtained crystals were observed first by optical microscopy and then by means of a Cambridge S360 scanning electron microscope equipped with a Oxford Inca energy 200 EDS system.

\section{Observed Growth Morphology}

From Solutions Supersaturated by $\mathrm{CO}_{2}$ Bubbling and Stripping. Highly supersaturated solutions were obtained by stripping $\mathrm{CO}_{2}$ from saturated $\mathrm{Li}_{2} \mathrm{CO}_{3}$ solution by means of $\mathrm{N}_{2}$ bubbling flow. In that case, $\mathrm{Li}_{2} \mathrm{CO}_{3}$ crystals were extremely elongated and showed a whiskerlike morphology that could not be indexed. At lower supersaturations, reached by slow reequilibration with the atmospheric carbon dioxide, crystal aggregates grew, formed by thin and sometimes twinned platelets not suitable for indexing.

By Evaporation of a Saturated Solution. In this case, single and 100 twinned crystals grew with sizes ranging from microto millimetric. Their [001] elongated morphology can be indexed and the occurrence of twins (either simple or polysynthetic) increases with the Titriplex III content in the growth solution.
Crystal grown under these conditions frequently are not wellterminated and show complex vicinal forms at the positive and negative outcrop of the [001] axis.

By Mixing of Two Solutions. When mixing sodium carbonate and lithium chloride solutions, high supersaturation values, and hence nucleation, are suddenly achieved. Then crystal size is too small to allow the indexing of crystallographic forms. However, if one mixes sodium hydrogen carbonate and lithium chloride, lower supersaturations are obtained, the mean crystal size increases, and indexing can be done.

By Dripping LiOH Solution into Carbonated Water.

Crystals are well-formed, with their morphology being comparable to those illustrated in images $\mathrm{a}$ and $\mathrm{b}$ in Figure 1, and we obtained evaporating $\mathrm{LiCl}: \mathrm{NaHCO}_{3}$ mixed solution without adding specific impurities. For the time being, the obtained nucleation frequency is still too high and, consequently, the mean size of crystals is too small.

In summary, the slow evaporation of saturated solutions (either pure or mixed) seems to represent a good compromise for obtaining large and well-shaped crystals (simple and twinned) suitable for a first approach on both growth morphology and surface patterns. Nevertheless, we think that dripping a $\mathrm{LiOH}$ solution into carbonated water is the most promising and right way to obtain high-quality and well-sized individuals (reducing the initial supersaturation by lowering the $\mathrm{LiOH}$ diffusion rate) to quantitatively determine the growth morphodromes. In fact, the mother solution does not in this case contain foreign substances (except water) that could interfere with crystal composition and shape; moreover, if one would investigate the effects induced by adding any impurity, new morphodromes could be obtained and compared with those resulting from pure solutions.

\section{Theoretical Equilibrium and Growth Morphology of the Single Crystal}

Building Units and PBCs in $\mathbf{L i}_{\mathbf{2}} \mathbf{C O}_{\mathbf{3}}$. The cell parameters and space group $(C 2 / c)$ we adopted in this study are those found in Effenberger and Zemann: ${ }^{12} a_{\mathrm{o}}=0.83593(36) \mathrm{nm} ; b_{\mathrm{o}}=$ $0.49725(11) \mathrm{nm} ; c_{\mathrm{o}}=0.61975(21) \mathrm{nm} ; \beta=114.83(3)^{\circ}$.

The single $\mathrm{Li}^{+}$and $\mathrm{CO}_{3}{ }^{2-}$ ions are the growth units (G.U.s) we considered coming from the mother phase. However, when a periodic bond chain $\left(\mathrm{PBC}^{11}\right)$ has to be found in the crystal, entire $\mathrm{Li}_{2} \mathrm{CO}_{3}$ groups (building units, B.U.s) have to be considered, taking into account all possible configurations of the B.U.s and ranking them according to the energy gained by their formation.

From the tetrahedral coordination of the lithium ion within the crystal structure, the hierarchy of the $\mathrm{Li}-\mathrm{O}$ bond length (nm) reads $p=0.1887 ; q=0.1952 ; r=0.1975$, and $s=0.2025$. 


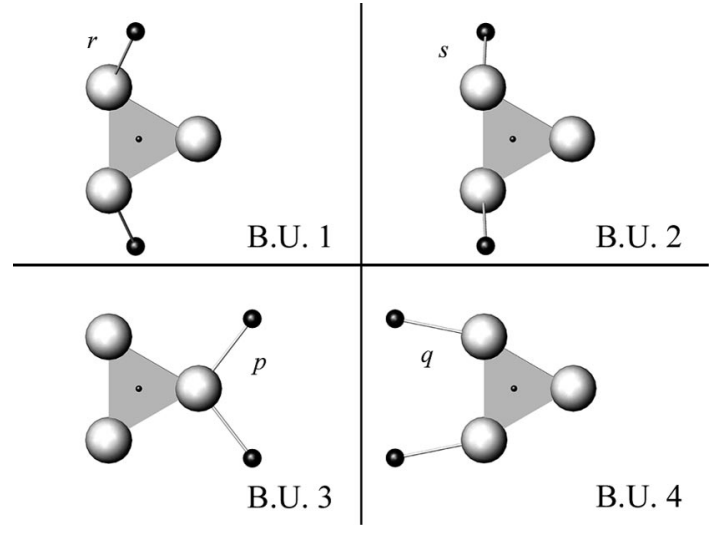

Figure 2. Four kinds of building units in the $\mathrm{Li}_{2} \mathrm{CO}_{3}$ structure, viewed along the [001] direction. $\mathrm{Li}$ atoms are the small black spheres. The different $\mathrm{Li}-\mathrm{O}$ bonds are labeled.

Accordingly, there are four different ways, illustrated in Figure 2, to choose the B.U.s, all of them having in common the same $\mathrm{CO}_{3}{ }^{2-}$ group. In B.U.1, B.U.2, B.U.3, and B.U.4, the carbonate ion is bonded to two lithium ions through $r, s, p$, and $q$ bonds, respectively. Each of these building units can be used as "molecules" to describe the structure of the crystal; then, each 3D cell of the crystal contains four equivalent B.U.s mutually related by the glide $c$ plane and by the translation vector $1 / 2\left(\tau_{1}+\tau_{2}\right)$.

In Table 1, the fractional coordinates of the atoms belonging to the B.U.s are collected; the labels we adopted differ from those by Effenberger and Zemann, simply to make the identification of each building unit easier.

The carbonate group is common to all four B.U.s. Its coordinates are as follows: $\mathrm{C}_{1} 0,0.0657,0.25 ; \mathrm{O}_{11} 0,0.3213$, $0.25 ; \mathrm{O}_{21} 0.1459,-0.0635,0.3127 ; \mathrm{O}_{31}-0.1459,-0.0635$, 0.1873 .

Character of the Main Crystal Forms. (a) $\{110\}$ Form. The $c$ plane generates a chain of $s$ bonds between the successive B.U.1 and of $r$ bonds between the successive B.U.2. The number of these bonds is four over the period $|[001]|=c_{\mathrm{o}}$. Because of the glide plane, the chain does not shown any resultant dipole moment perpendicular to its development axis. Thus, the $\mathrm{Li}_{2} \mathrm{CO}_{3}$ structure can be viewed as made by a 2D array of [001] PBCs, reproduced by the translation vectors $\tau_{1}, \tau_{2}$, and $1 / 2\left(\tau_{1}+\tau_{2}\right)$ and symbolically enclosed in elliptically shaped islands (Figure 3 ). Bonds among adjacent islands develop only along the equivalent [110] and [1̄0] directions. Both slices, having thickness $d_{110}=d_{1 \overline{1} 0}$, contain two PBCs, and then $\{110\}$ is a $F$ form. From the extinction rules imposed by the space group $C 2 / c$, the two other slices allowed for growth in this zone must have thicknesses $d_{200}$ and $d_{020}$, respectively. Because any bond can be found among islands along both the [010] and the [100] direction, $\{100\}$ and $\{010\}$ have to be considered as S (stepped) forms (Figure 3).
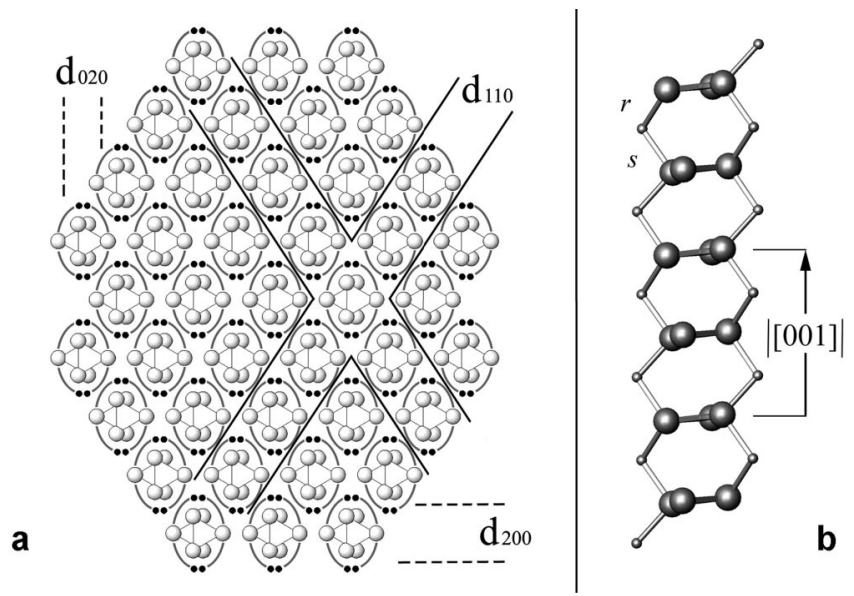

Figure 3. (a) [001] projection of the $\mathrm{Li}_{2} \mathrm{CO}_{3}$ structure. The [001] PBCs are enclosed in elliptically shaped islands. Bonds among adjacent islands develop only along the equivalent $\langle 110\rangle$ directions; both $d_{110}$ slices then contain two PBCs. Thus, the prism $\{110\}$ is an F form. The same PBCs are not linked within $d_{200}$ and $d_{020}$ slices (allowed by the extinction rules). Hence, the $\{100\}$ and $\{010\}$ forms show S character. (b) How the [001] PBC develops along its repeat period. B.U.1s are mutually linked by $s$ bonds along the $\mathrm{PBC}$ axis.

(b) $\{001\}$ Form. From a projection along one of the equivalent [110] and [1 10$]$ directions, $<110>$ chains of B.U.1s are found, bonded by $p$ and $q$ bonds, with a $\left[\begin{array}{lll}1 / 2 & 1 / 2 & 0\end{array}\right]$ period and within a $d_{002}$ slice (Figure 4$)$. These couple of chains are true PBC and give the $\mathrm{F}$ character to the $\{001\}$ form because the $d_{002}$ slice does not show dipole moment perpendicular to its surface, thanks to the $2_{1}$ axis parallel to [010] direction and lying in the middle of the slice. Further, starting from B.U.1, neither [010] PBC nor [100] PBC can be found within the $d_{002}$ slice because the corresponding chains can be constructed only by $\left[\begin{array}{lll}1 / 2 & 1 / 2 & 0\end{array}\right]$ segments of the $<110>$ PBCs.

(c) $\{10 \overline{1}\}$ Form. B.U.3s and B.U.4s are respectively bonded by couples of the $q$ and $p$ bonds related by the $2_{1}$ axis, so determining the [010] PBCs (Figure 5). In turn, these PBCs are bonded, within a $d_{002}$ slice, through $r$ bonds reproduced by the $2_{1}$ screw axis; this confirms the F character of the $\{001\}$ form. Moreover, it ensues that two successive [010] PBCs are bonded along the [101] direction, through a couple of $s$ bonds that generate the [101] PBC. Hence, because of the extinction rules, two families of PBCs run within a $\mathrm{d}_{\overline{2} 02}$ slice, so giving rise to the $\mathrm{F}$ character of the $\{\overline{1} 01\}$ form. No other $\mathrm{F}$ form can be deduced in the [010] zone. In fact, no bond can set up along the [001] direction and within a $d_{200}$ slice, according to the previous finding concerning the $S$ character of the $\{100\}$ form. Moreover, within a $\mathrm{d}_{\overline{1} 02}$ layer, the [010] PBCs are bonded by alternating $r$ and $s$ bonds but the space group rules impose to the $h 0 l$ layers the condition $h+l=2 n$ and then the thickness allowed for growth is $d_{2} 04$. Because no bond exists between the [010] PBCs within this half layer, $\{\overline{1} 02\}$ is an $\mathrm{S}$ form.

Table 1. Fractional Coordinates of the Lithium Atoms Belonging To the Four Building Units Describing the Structure of Lithium Carbonate

\begin{tabular}{|c|c|c|c|c|c|c|c|}
\hline \multicolumn{4}{|c|}{ B.U.1 } & \multicolumn{4}{|c|}{ B.U.2 } \\
\hline atom label & $x$ & $y$ & $z$ & atom label & $x$ & $y$ & $z$ \\
\hline $\operatorname{Li}_{11}(x, y, z)$ & -0.3035 & 0.0516 & 0.3344 & $\operatorname{Li}_{11}(x,-y, z-1 / 2)$ & -0.3035 & -0.0516 & -0.1656 \\
\hline $\operatorname{Li}_{21}(x, y, z)$ & 0.3035 & 0.0516 & 0.1656 & $\operatorname{Li}_{21}(x,-y, z+1 / 2)$ & 0.3035 & -0.0516 & 0.6656 \\
\hline \multicolumn{4}{|c|}{ B.U.3 } & \multicolumn{4}{|c|}{ B.U.4 } \\
\hline atom label & & $y$ & $z$ & atom label & $x$ & $y$ & $z$ \\
\hline $\operatorname{Li}_{11}(x+1 / 2, y+1 / 2, z)$ & & 0.5516 & 0.3344 & $\operatorname{Li}_{11}(x+1 / 2, y-1 / 2, z)$ & 0.1965 & -0.4484 & 0.3344 \\
\hline $\operatorname{Li}_{21}(x-1 / 2, y+1 / 2, z)$ & & 0.5516 & 0.1656 & $\mathrm{Li}_{21}(x-1 / 2, y-1 / 2, z)$ & -0.1965 & -0.4484 & 0.1656 \\
\hline
\end{tabular}



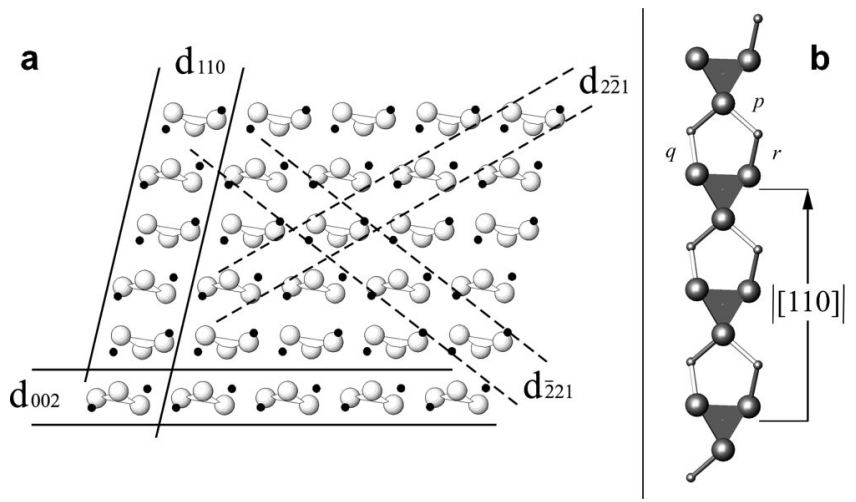

Figure 4. (a) [110] projection of the structure that shows [110] PBCs linked by $p$ and $q$ bonds within a $d_{002}$ slice and by $s$ bonds within a $d_{110}$ slice. It follows that the $\{001\}$ and the $\{110\}$ forms have $F$ character. On the contrary, the $\{\overline{2} 21\}$ and the $\{2 \overline{2} 1\}$ forms have $S$ character, [110] PBCs being not bonded within $d_{\overline{2} 21}$ and $d_{2 \overline{2} 1}$ slices, respectively. (b) The [110] PBC, seen along the [001] direction, is made by B.U.1s mutually linked by $p$ and $q$ bonds.
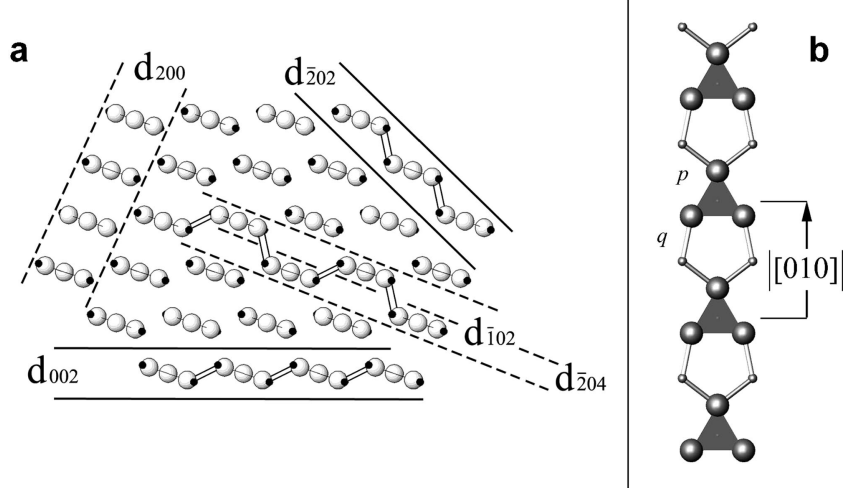

Figure 5. (a) [010] projection of the $\mathrm{Li}_{2} \mathrm{CO}_{3}$ structure. [010] PBCs are linked by $r$ bonds within a $d_{002}$ slice and by $s$ bonds within a $d_{\overline{2} 02}$ slice. Hence the $\{001\}$ and the $\{\overline{101}\}$ forms have $F$ character. The space group extinction rules allow the growth of $d_{\overline{2} 04}$ slices. Within this thickness, any bond exists among [010] PBCs, so the $\{\overline{1} 02\}$ is an S form. (b) How the [010] PBC develops along its repeat period, with B.U.3s being mutually linked by $q$ bonds along the PBC axis.

(d) $\{\overline{1} 11\}$ Form. From the projection along the [101] direction (Figure 6), it ensues that the $\{\overline{1} 01\}$ form confirms its $F$ character. Further, although the $\{010\}$ form looks like a stepped one because its B.U.s are bonded within a $d_{010}$ slice, the new $\{\overline{1} 11\}$ F form appears because $r$ bonds develop between [101] PBCs, within a $d_{\overline{1} 11}$ slice, along the [110] direction.

Surface and Attachment Energies. Once the qualitative information has been obtained to evaluate the most probable surface profiles of the flat faces, we calculated the energies of the surface sites relevant to equilibrium and growth. To do that, we developed a computer code CSEHP (crystal site energies by Hartman-Perdok method) whose potentialities have been already described ${ }^{13,14}$ The potential function we adopted is a simple one: the electrostatic charges we choose for the carbonate group are the same used for the evaluation of the equilibrium and growth forms of calcite, ${ }^{15}$ whereas the charge on the lithium ion recovers the electroneutrality. For the time being, we describe the energy between a couple of atoms $i$ and $j$, related by a distance $r_{i j}$ by the simple coulomb interaction: $E_{i j}=\left(q_{i} q_{j}\right)$ $r_{i j}$ ) where $q(\mathrm{Li})=0.93385 \mathrm{e}, q(\mathrm{C})=0.98530 \mathrm{e}$, and $q(\mathrm{O})=$ -0.9510 e. Moreover, we compare these results to those just
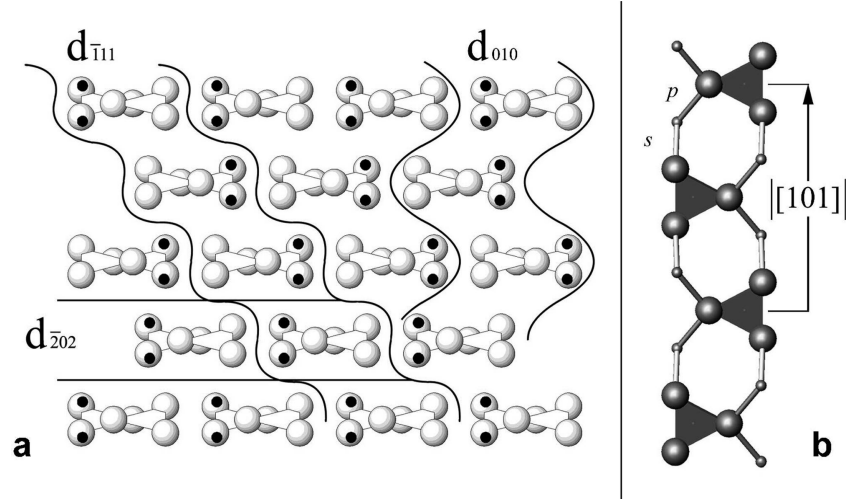

Figure 6. (a) [101] projection showing [101] PBCs linked by $q$ bonds within a $d_{\overline{2} 02}$ slice and by $r$ bonds within a $\mathrm{d}_{\overline{1} 11}$ slice. This confirms the $\mathrm{F}$ character of the $\{\overline{1} 01\}$ and of the $\{\overline{1} 11\}$ forms. The $\{010\}$ form behaves as a stepped one because its BUs are bonded within a $d_{010}$ slice, which is forbidden by the extinction rules. (b) The [101] PBC, seen along the [001], is made by B.U.3s mutually linked by $s$ bonds.

obtained in our research group through ab initio methods to better evaluate the surface energies of our crystal. ${ }^{16}$

As a first step, we calculated the formation energy of the building units. To do that, all the interaction energies between atoms belonging to each B.U. were calculated and then, as we did in a previous work, ${ }^{15}$ the formation energy of the $\mathrm{CO}_{3}{ }^{2-}$ group $\left(0.226 \times 10^{-10} \mathrm{erg}\right)$ was subtracted, because it was reasonably assumed that this group pre-exists in solution. The resulting energy formations of the four B.U.s, expressed in 1 $\times 10^{-10} \mathrm{erg}$, are $0.256,0.248,0.234$, and 0.224 for B.U.1, B.U.2, B.U.3, and B.U.4, respectively. The small difference between the highest and lowest energy values (less than 12\%) implies that all the B.U.s have more or less the same probability to occur and reflects the slightly distorted tetrahedral coordination of the lithium ion.

Surface energies $\gamma_{h k l}$ are calculated through the Born-Stern approximation: $\gamma_{\infty}(h k l)=\left(W_{h k l}^{\mathrm{sep}} / 2 A_{h k l}\right)$ where $W_{h k l}^{\mathrm{sep}} / 2 A_{h k l}$ represents the work at $0 \mathrm{~K}$ to be spent to separate the crystal along an infinite $h k l$ surface over the new generated $2 A_{h k l}$ area. We also calculated $E_{\text {att }}(h k l)$, the attachment energy of the $\{h k l\}$ form, which represents the energy released, per formula unit, when a slice of $d_{\mathrm{hkl}}$ thickness, allowed by the extinction rules, crystallizes on the $\{h k l\}$ surfaces. From the Hartman-Perdok theory, the growth rate of a given crystal form can be reasonably assumed proportional to its attachment energy, when solvent and impurity action are not considered. ${ }^{17}$ Both $\gamma_{\infty}(h k l)$ and $E_{\text {att }}(h k l)$ values are quoted in Table 2.

\section{Comparison between Experimental and Theoretical Morphologies, Growth of the (100) Twins, and Re-Entrant Angle Effect}

Equilibrium Shape (ES) of the Crystal and the Comparison between Experimental and Theoretical Growth Morphology. From the just-quoted values, both theoretical equilibrium and growth forms of the crystal can be drawn, as shown in Figure 7. Only three out of four F forms can belong to the ES, independently of the way of calculating the surface energies. Going into detail, the $\{001\}$ form turns out to be the most important one, as far as the calculus represents the most appropriate physical situation, whereas the prism $\{110\}$ behaves in the opposite way.

Among the calculated stepped forms, it is worthwhile to consider the peculiar $\{100\}$ interface. This form can assume 
Table 2. Specific Surface and Attachment Energies Calculated for the Main Forms of $\mathrm{Li}_{2} \mathrm{CO}_{3}$ Crystal

\begin{tabular}{|c|c|c|c|c|c|}
\hline \multirow[b]{2}{*}{$\mathrm{hkl}$} form & \multirow[b]{2}{*}{$\begin{array}{c}\text { face } \\
\text { character }\end{array}$} & \multicolumn{2}{|c|}{ from formal charge potential } & \multicolumn{2}{|c|}{ from ab initio calculation ${ }^{16}$} \\
\hline & & $\begin{array}{c}\text { unrelaxed surfaces } \\
\gamma_{\infty}\left(\mathrm{erg} \mathrm{cm}^{-2}\right) \\
\end{array}$ & $\begin{array}{c}\text { unrelaxed surfaces } \\
E_{\text {attachment }}\left(\mathrm{erg} \times 10^{-12} / \text { f.u. }\right) \\
\end{array}$ & $\begin{array}{c}\text { unrelaxed surfaces } \\
\gamma_{\infty}\left(\mathrm{erg} \mathrm{cm}^{-2}\right) \\
\end{array}$ & $\begin{array}{c}\text { relaxed surfaces } \\
\gamma_{\infty}\left(\mathrm{erg} \mathrm{cm}^{-2}\right) \\
\end{array}$ \\
\hline$\{001\}$ & $\mathrm{F}$ & 540 & 2.217 & 248 & 160 \\
\hline$\{\overline{1} 01\}$ & $\mathrm{F}$ & 496 & 2.209 & 392 & 260 \\
\hline$\{110\}$ & F & 993 & 2.786 & 697 & 590 \\
\hline$\{111\}$ & $\mathrm{F}$ & 2178 & 6.716 & & \\
\hline$\{100\}$ from B.U.1 & S & 3383 & 10.670 & & \\
\hline
\end{tabular}

two profiles, according to whether its main $\mathrm{PBC}$ was derived from BU.1 or BU.3 (Figures 3 and 5a). In the first case, the profile is unduly attributable to the shape of the [001] PBC, whereas the (100) flattens when obtained from the [010] PBC. Correspondingly, the surface energy varies from $\gamma_{100}(\mathrm{BU} 1)=$ 3383 to $\gamma_{100}(\mathrm{BU} 3)=1959 \mathrm{erg} \mathrm{cm}^{-2}$, respectively. Neither of these profiles can enter in the theoretical ES of the crystal; however, a $10 \%$ reduction of the lowest surface tension value could allow the $\{100\}$ form to intersect the $\{110\}$ form. Such a situation could realize at the crystal/solution equilibrium, because the surface tension lowering due to water adsorption is surely higher on the (100) stepped face, composed by elementary segments of (110) profile, than on the (110) flat one. This means that the $\{100\}$ form can enter the equilibrium crystal shape in solution and then can play the important role of the original composition surface ${ }^{18}$ for contact twinning at nucleation and during growth, as we will see later on.

If the ES of a crystal can be observed only under well-defined conditions, ${ }^{19}$ the GS is that we usually observe and then can compare with the theoretical one. In our case and when confining our attention to the GS of the single crystal, the experimental GS of crystal grown in solutions containing only the species building up the crystal and water is that illustrated in Figure 1a. The occurring forms are those predicted and shown in Figure 7. However, at variance with the theoretical GS, Figure 7, which is representative of the observed crystals, shows a very small face, vicinal of the $\{010\}$ form, and that the crystal is strongly [001] elongated. We are not able to distinguish if this difference could be due to a kinetic effect and/or to the difference in water adsorption on the involved $\mathrm{F}$ forms. The full understanding of that is out of the aim of the present paper; the time-consuming work on the dependence of the $\mathrm{Li}_{2} \mathrm{CO}_{3}$ morphology on initial supersaturation in pure medium is the subject of future research.

(a) 100 Twin Law and the Original Composition Plane 100. According to Mallard ${ }^{2}$ and Zemann, ${ }^{20}$ the $\mathrm{Li}_{2} \mathrm{CO}_{3}$ crystals very

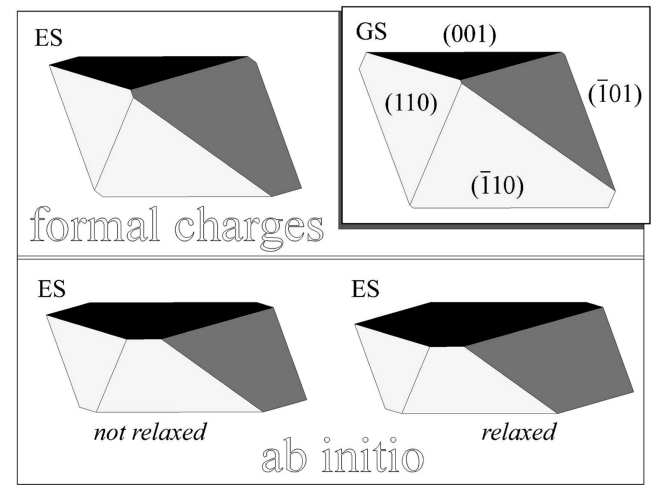

Figure 7. Equilibrium shape (ES) of the $\mathrm{Li}_{2} \mathrm{CO}_{3}$ crystal evaluated by means of formal charges potential and from ab initio calculation. The theoretical growth shape (GS) is evaluated from attachment energies calculated by formal charge potential. often exhibit 100 contact twins. Zemann explained the high twinning frequency through the pseudo-orthorhombic character of the $\mathrm{Li}_{2} \mathrm{CO}_{3}$ structure. In fact, there is a simple relation between the pseudo-orthorhombic cell and the original monoclinic one: $a_{\text {ortho }}=\mid[201]_{\text {mono }} ; b_{\text {ortho }}=b_{\text {mono }} ; c_{\text {ortho }}=c_{\text {mono }} ; \alpha_{\text {ortho }}$ $=\gamma_{\text {ortho }}=90^{\circ} ; \beta_{\text {ortho }}=93.07^{\circ}$.

Moreover, the structural continuity across the twinned interface is assured, because the [101] PBC of the parent crystal continues with the [100] direction of the twinned one, as may be seen in Figure 8, where the simulated structure of a 100 twin is projected along the [010] direction. We will discuss, in the following, the 100 law for the simple twins and its consequences on the twin morphology.

Let us now consider that both the twin operation and the original composition plane of the twin are represented by the 100 plane. Remembering that $\{100\}$ is a stepped form, we have to imagine that a "twinned" [001] PBC should form coherently with the scheme drawn in Figure 3a. The coordinates of the atoms placed in twin position and referred to the crystallographic frame of the parent crystal are then generated by a 100 mirror placed at a distance $d_{100}$ with respect to the origin, through the transformation

$$
x_{\mathrm{T}}=2 a_{\mathrm{o}}-x_{\mathrm{P}} ; \quad y_{\mathrm{T}}=y_{\mathrm{P}} ; \quad z_{\mathrm{T}}=z_{\mathrm{P}}-2\left(a_{\mathrm{o}}-x_{\mathrm{P}}\right) \cos \beta
$$

where the origin of the coordinates is kept in the center of the parent [001] PBC and the suffixes T and P refer to the twinned and parent crystal, respectively.

The transformation leaves more or less unchanged the inclination of the carbonate groups with respect to the [001]

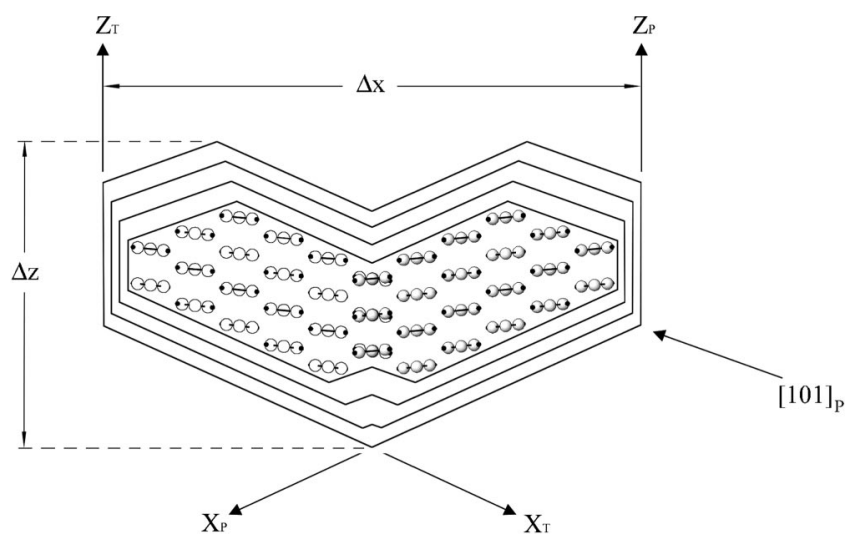

Figure 8. Structure of a twinned $\mathrm{Li}_{2} \mathrm{CO}_{3}$ nucleus viewed along the [010] direction, which is common to both parent $(\mathrm{P})$ and twinned $(\mathrm{T})$ individuals. The initial twin profile is calculated from the relaxed values of the surface tensions (see text and Table 2). It is worth noting the small misalignment of the growth units at the twin interface and that the structural continuity of the twin is assured by the parallelism of the $X$ axis of the twinned individual and of the [101] direction of the parent one. The varying profiles represent the evolution steps of the twin shape and take into account the growth acceleration due to the reentrant angle effect. 
axis, because they are nearly perpendicular to it, as is seen from Figure 8. Nevertheless, the pure 100 reflection is not sufficient to move the twinned atoms toward their minimum potential energy. To do that, one should glide the twinned PBC by $-0.02558 \mathrm{~nm}$ along the [001] direction. After such a translation, the twinned lithium atoms facing the parent crystal reach exactly the same position they had in it. When considering now the new distances among first and second neighbors, we may say that (1) all $8 \mathrm{Li}-\mathrm{Li}$ distances remain unchanged, (2) the same situation occurs for $12 \mathrm{Li}-\mathrm{O}$ distances, (3) $24 \mathrm{O}-\mathrm{O}$ distances result in being modified along with $16 \mathrm{Li}-\mathrm{O}$ distances. This means that two-thirds of the interactions are modified at the interface between the twinned PBC and the parent crystal.

When assuming the simple Coulomb interaction energy $W$ $=q_{1} q_{2} / 4 \pi \epsilon r$ between two charges $q_{1}$ and $q_{2}$ at a distance $r$ apart in a medium of permittivity $\epsilon$ and neglecting the contribution of the carbon atoms, we calculated, per unit segment of the [001] PBC, the following differences between twinned and parent positions: (1) the attractions are slightly lowered $(-0.218 \%),(2)$ the repulsions are increased $(+2.165 \%)$.

Having considered that one-third of the interactions are unmodified, one can reasonably say that the potential energy of the faulted twinned interface is lowered only by the $2 \%$ with respect to the perfect one, which explains very well the high occurrence frequency of the contact 100 twin of the $\mathrm{Li}_{2} \mathrm{CO}_{3}$ crystals, even at low supersaturation values, and that the growth mechanism of the twinned individuals preferentially occurs trough a faulted 1D nucleation along the [001] PBC.

(b) Growth Morphology of the 100 Twins and the Re-entrant Angle Effect. Once the twin nucleation is achieved, the final twin shape will depend, obviously, on temperature and supersaturation but also on its initial geometry at the composition interface. From the observation, it follows that $\mathrm{Li}_{2} \mathrm{CO}_{3}$ twins show equally sized parent and twinned parts. It can then be reasonably induced that this kind of twins experienced a $3 \mathrm{D}$ twinned nucleation ${ }^{21}$ expressed by two identical embryos born in contact, since the beginning, on their common (100) face. Both size and shape of this twinned nucleus are fixed by the thermodynamic supersaturation $\Delta \mu=k_{\mathrm{B}} T \ln \beta$ and by the specific surface tensions of their $\{h k l\}$ forms, respectively. Here, $k_{\mathrm{B}}$ and $T$ represent the Boltzmann constant and the absolute temperature, whereas $\beta=a / a_{\mathrm{eq}}$ is the supersaturation ratio depending on the actual and equilibrium activities of the solution. Let us consider the initial twin shape is that shown in Figure 8, where the calculated twin perimeter of the [010] projection depends on the relaxed values of the specific surface energies quoted in Table 2. Only the extension of the initial 100 common interface is arbitrarily assumed, because the (100) face cannot belong to the equilibrium crystal shape, as discussed earlier.

Nevertheless, this interface must exist, otherwise the observed contact twins could not even form. Figure 8 shows that two dihedral re-entrant angles forms at the two opposite outcrops of the contact plane: one of them is between the (001) faces and the other between the $(\overline{101})$ ones. Once the twin shape at nucleation was drawn, we evaluated its successive growth stages by considering the growth rate of the faces proportional to their respective $E_{\text {att }}$ values (i.e., assuming the Hartman ${ }^{17}$ relationship $\left.\mathrm{R}_{h k l} \propto E_{h k l}^{\mathrm{att}}\right)$ and remembering that the re-entrant angle effect ${ }^{22}$ accelerates the growth rate owing to the easier occurring $1 \mathrm{D}$ and/or 2D nucleation where the two faces meet. This explains, on one side, the increasing asymmetry between the growth rate of the twin along the opposite directions of the [001] axis and, on the other side, the usually observed [001] elongated shape of $\mathrm{Li}_{2} \mathrm{CO}_{3}$ twins. As a matter of fact, the twin enlargement $(\Delta x)$ in the $(010)$ cross section is determined by $2.18 \times R_{110}$, because of both twin geometry and advancement rate of the $\{110\}$ form, whereas the twin elongation $(\Delta z)$ is at least equal to $1.82 \times\left(R_{\overline{1} 01}+R_{001}\right)$. Hence, having considered the values quoted in Table 2 and the acceleration due to the re-entrant angle effect, one obtains that $\Delta z \geq 1.66 \Delta x$ is the condition ruling the elongation ratio of our contact twins.

Moreover, once the re-entrant angle has been filled along the

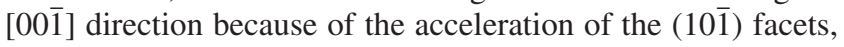
the twins assume the swallow-tail shape as first observed by Mallard. ${ }^{2}$ Finally, an unambiguous example of the filling up operating in the re-entrant angles is shown in Figure $1 \mathrm{~b}$ where the dihedral angle of $112^{\circ}$ made by the (110) parent and (110) twinned faces is progressively filled since the beginning of growth, so giving rise to the sharp and long $\mathrm{V}$-shaped groove.

\section{Conclusions}

Experimental and theoretical growth morphologies of monoclinic $\mathrm{Li}_{2} \mathrm{CO}_{3}$ crystal have been compared in light of the Hartman-Perdok theory. The theoretical equilibrium shape of the crystal has also been calculated at $0 \mathrm{~K}$ and with respect to a vacuum using a formal charge potential function and compared with that obtained from surface energy values evaluated by ab initio calculation. Theoretical equilibrium and growth shapes show a very simple morphology composed only by three $\mathrm{F}$ forms, $\{001\},\{\overline{1} 01\}$, and $\{110\}$, which are also the dominant forms of the observed morphology of crystals grown in pure aqueous solutions. Thus, it ensues that the poor lithium carbonate morphology strikingly differs from the rich morphology of orthorhombic carbonates (aragonite-like) and the extremely variable one of the rhombohedral carbonates (calcite-like).

Two main discrepancies come out from the comparison between observation and theoretical prediction. The first one concerns the $\{100\}$ form that does not appear both on theoretical equilibrium and growth shape of the crystal, even if it plays an essential role in nucleation and growth of the commonly occurring 100 contact twins; the second one is related to the characteristic [001] elongation of single crystals, which is in contrast with the more isotropic theoretical growth shape.

It is likely that considering water adsorption could allow us to face the first drawback, whereas carrying out growth experiments at constant supersaturation in pure aqueous solution could help to overcome the second difficulty.

As far as the twin growth is concerned, we succeeded in explaining how can form the observed peculiar shapes of contact twins thanks to the equilibrium shape at nucleation and to the re-entrant angle kinetic effect.

Starting from the knowledge acquired in this work on the $\mathrm{Li}_{2} \mathrm{CO}_{3}$ surfaces treated as though they were in equilibrium with the vacuum, we will deal in the near future with a new potential function fitted with the just considered ab initio results and will adopt it both to simulate the adsorption of water molecules on the most important $\mathrm{Li}_{2} \mathrm{CO}_{3}$ faces and to explain how the $\mathrm{Li}_{2} \mathrm{CO}_{3}$ surface structures adapt so well to the different calcite surfaces, as it comes out from the observed epitaxies.

Finally, the features we found on the contact twins will be fundamental to understanding the monoclinic and the pseudotrigonal polysynthetic twins epitaxially grown on the calcite rhombohedron that we will deal with in a forthcoming paper. ${ }^{23}$

Acknowledgment. We thank Dr. Marco Bruno and Dr. Mauro Prencipe (Università degli Studi) for useful discussion on calculations. This work was supported by Ministero 
dell'Istruzione, Università e Ricerca (MIUR), with funds related to PRIN 2005.

\section{References}

(1) Reeder, R. J. In Carbonates: Mineralogy and Chemistry; Reviews in Mineralogy; Reeder, R. J. Ed.; Mineralogical Society of America: Chantilly, VA, 1990; Vol. 11, Chapter 1, pp 1-47.

(2) Mallard, E. Bull. Soc. Fr. Minéral. 1892, 15, 21-26.

(3) Zheng, M.; Liu, W. Acta Mineral. Sin. 1987, 7, 221-226 (in Chinese).

(4) Anderson, A. J.; Clark, A. H.; Gray, S. Can. Mineral. 2001, 39 (6), $1513-1527$.

(5) Rajam, S.; Mann, S. J. Chem. Soc., Chem. Commun. 1990, 17891791.

(6) Nefyodova, I. V.; Lyutin, V. I.; Borodin, V. L.; Chvanski, P. P.; Leonyuk, N. I. J. Cryst. Growth 2000, 211, 458-460.

(7) Kenway, P. R.; Oliver, P. M.; Parker, S. C.; Sayle, D. C.; Sayle, T. X. T.; Titiloye, J. O. Mol. Simul. 1992, 9, 83-89.

(8) Parker, S. C.; Kesley, E. T.; Oliver, P. M.; Titiloye, J. O. Faraday Discuss. 1993, 95, 75-84.

(9) Pastero, L.; Costa, E.; Bruno, M.; Rubbo, M.; Sgualdino, G.; Aquilano, D. Cryst. Growth Des. 2004, 4, 485-490.
(10) Pastero, L.; Aquilano, D.; Costa, E.; Rubbo, M. J. Cryst. Growth 2005, 275, e1625-e1630.

(11) Hartman, P. In Crystal Growth: An Introduction; Hartman, P., Ed.; North-Holland: Amsterdam, 1973; pp 367-402.

(12) Effenberger, H.; Zemann, J. Z. Kristallogr. 1979, 150, 133-138.

(13) Aquilano, D.; Rubbo, M.; Catti, M.; Pavese, A. J. Cryst. Growth 1997, 182, 168-184.

(14) Rubbo, M.; Aquilano, D. J. Cryst. Growth 1997, 194, 156-159.

(15) Aquilano, D.; Calleri, M.; Natoli, E.; Rubbo, M.; Sgualdino, G. Mater. Chem. Phys. 2000, 66, 159-163.

(16) Bruno, M., Prencipe, M. Surf. Sci. 2007, 601, 3012-3019.

(17) Hartman, P.; Bennema, P. J. Cryst. Growth 1980, 49, 145-156.

(18) Hartman, P. Z. Kristallogr. 1956, 107, 225-237.

(19) Kern, R. In Morphology of Crystals (Part A); Sunagawa, I., Ed.; Terra Scentific Publishing: Tokyo, 1987; pp 77-206.

(20) Zemann, J. Acta Crystallogr. 1957, 10, 664-666.

(21) Simon, B. Thesis, UniversityAix-Marseille, Aix-en-Provence, France, 1968.

(22) Boistelle, R.; Aquilano, D. Acta Crystallogr., Sect. A 1977, 33, 642648

(23) Massaro, F. R.; Pastero, L.; Costa, E.; Aquilano, D. 2008, in preparation. CG070199S 